T. Ono

Nagoya Math. J.

Vol. 79 (1980), 131-140

\title{
ON THE GROUP OF AUTOMORPHISMS OF A HOPF MAP
}

\author{
TAKASHI ONO
}

\section{§1. Introduction}

Let $K$ be an infinite field of characteristic not 2. Let $q_{X}, q_{Y}$ be nonsingular quadratic forms on vector spaces $X, Y$ over $K$, respectively. Assume that there is a bilinear map $B: X \times Y \rightarrow Y$ such that $q_{Y}(B(x, y))=q_{X}(x) q_{Y}(y)$. To each such triple $\left\{q_{X}, q_{Y}, B\right\}$ one associates the Hopf map $h: Z=X \times Y$ $\rightarrow W=K \times Y$ by $h(z)=\left(q_{X}(x)-q_{Y}(y), 2 B(x, y)\right), z=(x, y)$. Denote by $q_{z}$, $q_{W}$ quadratic forms on $Z, W$, respectively, defined by $q_{z}(z)=q_{X}(x)+q_{Y}(y)$, $q_{W}(w)=u^{2}+q_{Y}(v), w=(u, v)$. One sees easily that $q_{W}(h(z))=q_{z}(z)^{2}$, which means that $h$ sends a sphere into a sphere. We shall denote by $G$ the group of automorphisms of $h$, i.e. the group formed by all automorphisms $s \in G L(Z)$ such that $h(s z)=h(z)$ for all $z \in Z$. After the model of the relationship of quadratic forms and orthogonal groups, it is natural to ask questions such as: what is the structure of $G$, how $G$ acts on the fibre, what the 1st cohomology of $G$ looks like, and how about the Hasse principle for $G$ when the ground field is a number field? In the present paper, we shall limit our considerations to the case where $X$ is an algebra with 1 over $K$ together with a nonsingular quadratic form $q_{x}$ such that $q_{x}(x y)$ $=q_{X}(x) q_{X}(y), x, y \in X$. Thanks to a theorem due to A. Hurwitz, such algebras, called composition algebras, are completely determined (cf. [1], Theorem 3.25, p. 73). Namely, an algebra $\left(X, q_{X}\right)$ is one of the following: (I) $X=K$; (II) $X=K+K$; (III) $X=$ a quadratic extension of $K$; (IV) $X$ = a quaternion algebra over $K$; (V) $X=$ a Cayley algebra over $K$. Furthermore, if $X=K$, then $q_{X}(x)=x^{2}$; otherwise $q_{X}$ is the norm form on $X$. Except for some easy arguments which work for an arbitrary triple $\left\{q_{X}\right.$, $\left.q_{Y}, B\right\}$, our results depend on the above theorem of Hurwitz. One can answer completely the questions mentioned above. For the general case, I have, at present, no definite idea how to handle it except the feeling that one needs detailed study of representations of Clifford algebras.

Received March 20, 1979. 


\section{§2. The subgroup $N$}

Notations being as in $\S 1$, since we have $q_{W}(h z)=q_{z}(z)^{2}$, for $s \in G$, we have $q_{Z}(s z)=e(s, z) q_{z}(z)$ with $e(s, z)= \pm 1$. Let $E=\left\{z \in Z, q_{z}(z) \neq 0\right\}$. Since $E$ is a non-empty open subset of the irreducible set $Z, E$ is also irreducible. Let $f_{s}$ be a function $E \rightarrow K$ defined by $f_{s}(z)=q_{z}(s z) / q_{z}(z)$. As $f_{s}$ is a continuous map, its image which is a subset of $\{ \pm 1\}$ must be irreducible, and so $e(s, z)=\chi(s)$, a function of $s$ only.*) Obviously, $\chi(s)$ is a homomorphism of $G$ into $\{ \pm 1\}$. Call $N$ the kernel of $\chi$. In this section, we consider $N$. Later on, we shall study the complement $G-N$ to decide whether $G=N$ or $[G: N]=2$.

An endomorphism $s: Z \rightarrow Z$ can be written as

$$
s=\left(\begin{array}{ll}
\alpha & \beta \\
\gamma & \delta
\end{array}\right) \quad \text { where } \alpha, \beta, \gamma, \delta
$$

are linear maps $X \rightarrow X, Y \rightarrow X, X \rightarrow Y, Y \rightarrow Y$, respectively. Using the column notation for $z=(x, y)$, we have

$$
s(z)=\left(\begin{array}{ll}
\alpha & \beta \\
\gamma & \delta
\end{array}\right)\left(\begin{array}{l}
x \\
y
\end{array}\right)=\left(\begin{array}{l}
\alpha x+\beta y \\
\gamma x+\delta y
\end{array}\right) .
$$

Now, we have

$$
s \in N \Longleftrightarrow h(s z)=h(z) \text { and } q_{z}(s z)=q_{z}(z) .
$$

In other words, we have

$$
s \in N \Longleftrightarrow\left\{\begin{array}{l}
q_{X}(\alpha x+\beta y)-q_{Y}(\gamma x+\delta y)=q_{X}(x)-q_{Y}(y), \\
q_{x}(\alpha x+\beta y)+q_{Y}(\gamma x+\delta y)=q_{X}(x)+q_{Y}(y), \\
B(\alpha x+\beta y, \gamma x+\delta y)=B(x, y),
\end{array}\right.
$$

or

$$
s \in N \Longleftrightarrow\left\{\begin{array}{l}
q_{X}(\alpha x+\beta y)=q_{X}(x), \\
q_{Y}(\gamma x+\delta y)=q_{Y}(y), \\
B(\alpha x+\beta y, \gamma x+\delta y)=B(x, y) .
\end{array}\right.
$$

Let $(,)_{X},(,)_{Y}$ be the inner product associated to $q_{X}, q_{Y}$, respectively. Then, (2.1) can be written as

$$
q_{X}(\alpha x)+q_{Y}(\beta y)+2(\alpha x, \beta y)_{X}=q_{X}(x) .
$$

*) We assumed the field $K$ infinite because we needed the Zariski topology here. 
Similarly, (2.2) can be written as

$$
q_{Y}(\gamma x)+q_{Y}(\delta y)+2(\gamma x, \delta y)_{Y}=q_{Y}(y) .
$$

If we put $x=0$ in (2.1), then we have $q_{x}(\beta y)=0$. If, on the other hand, we put $y=0$ in (2.1), then we have $q_{x}(\alpha x)=q_{x}(x)$ and hence $\alpha \in O\left(q_{x}\right)$, the orthogonal group of $q_{X}$. Substituting these results back in (2.4), we see that $(\alpha x, \beta y)_{X}=0$ for all $x \in X, y \in Y$. Since $\alpha$ is invertible, this implies that $\beta y=0$ for all $y$, i.e. $\beta=0$. Similarly, using (2.2), (2.5), we see that $\gamma=0$ and $\delta \in O\left(q_{Y}\right)$. We have therefore proved that

$$
N=\left\{s=\left(\begin{array}{ll}
\alpha & 0 \\
0 & \delta
\end{array}\right), \alpha \in O\left(q_{x}\right), \delta \in O\left(q_{Y}\right), B(\alpha x, \delta y)=B(x, y)\right\}
$$

(2.7) Remark. Let $e$ be a vector in $X$ such that $q_{X}(e)=1$. If such $e$ is available, the map $t$ defined by $t(y)=B(e, y)$ belongs to $O\left(q_{Y}\right)$ in view of the relation $q_{Y}(B(x, y))=q_{X}(x) q_{Y}(y)$. Therefore, if we put $B_{0}(x, y)=t^{-1} B(x, y)$, then we get a bilinear map $X \times Y \rightarrow Y$ with the property $B_{0}(e, y)=y, y \in$ $Y$, in addition to the property $q_{Y}\left(B_{0}(x, y)\right)=q_{X}(x) q_{Y}(y)$. Hence, without much loss of generality, we may assume from the beginning that the bilinear map $B$ satisfies the condition that $B(e, y)=y$ for an $e \in X$ with $q_{X}(e)$ $=1$. It then follows that $\delta y=B(e, \delta y)=B\left(\alpha^{-1} e, y\right)$ and so we have a group isomorphism:

$$
N \approx\left\{\alpha \in O\left(q_{x}\right), B\left(\alpha x, B\left(\alpha^{-1} e, y\right)\right)=B(x, y)\right\} .
$$

\section{§3. The set $G-N$}

First of all, we have

$$
s \in G-N \Longleftrightarrow h(s z)=h(z) \text { and } q_{z}(s z)=-q_{z}(z) .
$$

Therefore,

$$
s \in G-N \Longleftrightarrow\left\{\begin{array}{l}
q_{X}(\alpha x+\beta y)-q_{Y}(\gamma x+\delta y)=q_{X}(x)-q_{Y}(y), \\
q_{X}(\alpha x+\beta y)+q_{Y}(\gamma x+\delta y)=-q_{X}(x)-q_{Y}(y), \\
B(\alpha x+\beta y, \gamma x+\delta y)=B(x, y),
\end{array}\right.
$$

or

$$
s \in G-N \Longleftrightarrow\left\{\begin{array}{l}
q_{x}(\alpha x+\beta y)=-q_{Y}(y) \\
q_{Y}(\gamma x+\delta y)=-q_{x}(x) \\
B(\alpha x+\beta y, \gamma x+\delta y)=B(x, y)
\end{array}\right.
$$


Here (3.1) can be written as

$$
q_{X}(\alpha x)+q_{X}(\beta y)+2(\alpha x, \beta y)_{X}=-q_{Y}(y)
$$

and (3.2) can be written as

$$
q_{Y}(\gamma x)+q_{Y}(\delta y)+2(\gamma x, \delta y)_{Y}=-q_{X}(x) .
$$

If we put $y=0$ in (3.1), we have $q_{X}(\alpha x)=0$. If we put $x=0$ in (3.1), we have $q_{X}(\beta y)=-q_{Y}(y)$ and hence $\beta$ is injective, i.e. $\beta$ embeds $\left(Y-q_{Y}\right)$ into $\left(X, q_{X}\right)$. Similarly, from (3.2), (3.5), $q_{Y}(\delta y)=0$ and $q_{Y}(\gamma x)=-q_{X}(x)$ where the latter implies that $\gamma$ embeds $\left(X, q_{X}\right)$ into $\left(Y,-q_{Y}\right)$. In other words, $\beta$ and $\gamma$ are isometries of $\left(X, q_{X}\right)$ and $\left(Y,-q_{Y}\right)$. Since (3.4) implies $(\alpha x, \beta y)_{X}$ $=0$ for all $x \in X, y \in Y$, we have $\alpha x=0$, i.e. $\alpha=0$. Similarly, by (3.5), we have $\delta=0$. We have therefore proved that

$$
\begin{array}{r}
G-N=\left\{s=\left(\begin{array}{ll}
0 & \beta \\
\gamma & 0
\end{array}\right), q_{X} \beta=-q_{X}, q_{Y} \gamma=-q_{X},\right. \\
B(\beta y, \gamma x)=B(x, y)\} .
\end{array}
$$

(3.7) Remark. As in (2.7), assume that $B$ satisfies the additional condition $B(e, y)=y$. Then we have $\gamma x=B(e, \gamma x)=B\left(x, \beta^{-1} e\right)$ and so a bijection of sets:

$$
G-N \approx\left\{\beta,\left(Y,-q_{Y}\right) \stackrel{\beta}{\longrightarrow}\left(X, q_{X}\right), B\left(\beta y, B\left(x, \beta^{-1} e\right)\right)=B(x, y)\right\} .
$$

(3.9) Remark. As (3.6) shows, we have $G=N$ unless $\left(X, q_{X}\right)$ and $\left(Y,-q_{Y}\right)$ are isometric. For example, if $\operatorname{dim} X \neq \operatorname{dim} Y$, then $G=N$, always. One can also determine the structure of the triple $\left\{q_{X}, q_{Y}, B\right\}$ with isometries

$$
\left(X, q_{X}\right) \underset{\beta}{\stackrel{r}{\rightleftarrows}}\left(Y,-q_{Y}\right)
$$

by making use of the theorem of Hurwitz.

\section{§4. Composition algebras}

Let $\left(X, q_{X}\right)$ be a composition algebra over $K$. This is a special case of the triple $\left\{q_{X}, q_{Y}, B\right\}$ where $Y=X, q_{Y}=q_{X}, B(x, y)=x y$. Moreover, $X$ has the identity 1 and so the remarks (2.7), (3.7) are available. If we put $a=\alpha^{-1}(1), b=\delta^{-1}(1)$, the last equality of (2.6) implies that $\alpha x=x b$ and $\delta y=a y$. Substituting these in (2.8), we have the group isomorphism: 


$$
\begin{aligned}
N & \approx\left\{a \in X, q_{X}(a)=1,\left(x a^{-1}\right)(a y)=x y \text { for all } x, y \in X\right\} \\
& =\left\{a \in X, q_{X}(a)=1, x(a y)=(x a) y \text { for all } x, y \in X\right\}
\end{aligned}
$$

If the algebra $X$ is associative, then we have simply:

$$
N \approx\left\{a \in X, q_{X}(a)=1\right\},
$$

the group of norm one in $X$. In view of the theorem of Hurwitz, it remains the case where $X$ is the Cayley algebra. In this case, we have $X=Y+$ $Y \omega, Y=K+K i+K j+K k$, a quaternion algebra. The multiplication and conjugation in $X$ are given as follows: $\omega^{2}=\mu \in K^{\times}, \omega x=\bar{x} \omega, x(y \omega)=(y x) \omega$, $(x \omega) y=(x \bar{y}) \omega,(x \omega)(y \omega)=\mu \bar{y} x, \overline{x+y \omega}=\bar{x}-y \omega, x, y \in Y$. Now, write $a=$ $b+c \omega, b, c \in Y$ and put $x=i, y=j$ in the relation $x(a y)=(x a) y$. Then, we end up with the equality $i b j-c k \omega=i b j+c k \omega$, which implies that $c$ $=0$, i.e. $a=b \in Y$. Next, put $x=y \omega, y=\omega$ in $x(a y)=(x a) y$. It then follows that $a \in K$, the center of $Y$. We have therefore proved that

$$
N \approx\{ \pm 1\} \quad \text { when } X \text { is a Cayley algebra . }
$$

We now turn to the set $G-N$. If we put $a=\gamma^{-1}(1), b=\beta^{-1}(1)$, the last equality of (3.6) implies that $\gamma x=x b, \beta y=a y$. Note that $q_{X}(a)=q_{X}(b)$ $=-1$ because $q_{x} \beta=-q_{x}=q_{x} \gamma$. Substituting these in (3.8), we have the bijection of sets:

$$
\begin{aligned}
G-N & \approx\left\{a \in X, q_{X}(a)=-1,(a y)\left(x a^{-1}\right)=x y \text { for all } x, y \in X\right\} \\
& =\left\{a \in X, q_{X}(a)=-1,(a y) x=(x a) y \text { for all } x, y \in X\right\} .
\end{aligned}
$$

If the algebra $X$ is commutative, then we have simply:

$$
G-N \approx\left\{a \in X, q_{X}(a)=-1\right\} \text {. }
$$

In view of the theorem of Hurwitz, it remains the cases where $X$ is a quaternion algebra or a Cayley algebra. Putting $y=1$ in the relation (ay) $x=(x a) y$, we see that $a \in K$ because these algebras are central. But then we must have $x y=y x$ if there is an $a \in K$ such that $q_{x}(a)=a^{2}=-1$. Thus the set $G-N$ is empty, i.e.

(4.6) $\quad G=N$ when $X$ is either a quaternion algebra or a Cayley algebra.

From (4.1)-(4.6), we get the following

(4.7) Theorem. Let $K$ be an infinite field of characteristic not $2, X$ be a composition algebra over $K$ and $n(x)$ be the norm form on $X$. Let $h: Z=$ $X \times X \rightarrow W=K \times X$ be the Hopf map given by $h(z)=(n(x)-n(y), 2 x y)$, 
$z=(x, y), G$ be the group of automorphisms $s \in G L(Z)$ such that $h(s z)=h(z)$ and $N$ be the subgroup of $G$ consisting of $s$ such that $q_{z}(s z)=q_{z}(z)$, where $q_{z}(z)=n(x)+n(y)$. According to the theorem of Hurwitz, classify $X$ as (I) $X=K$; (II) $X=K+K$; (III) $X=a$ quadratic extension of $K$; (IV) $X$ a quaternion algebra over $K$; (V) $X=a$ Cayley algebra over $K$. Then we have the following table:

\begin{tabular}{c|c|ll}
\hline type of $X$ & $N$ & \multicolumn{1}{|c}{$[G: N]$} \\
\hline (I) & $\{ \pm 1\}$ & 1 & $\begin{array}{l}\text { when } \sqrt{-1} \notin K \\
\text { when } \sqrt{-1} \in K\end{array}$ \\
\hline (II) & $K^{\times}$ & 2 & \\
\hline (III) & $\{x \in X, n(x)=1\}$ & $\begin{array}{l}1 \text { when } n(x)=-1 \text { has no solutions } \\
2 \text { when } n(x)=-1 \text { has a solution }\end{array}$ \\
\hline (IV) & $\{x \in X, n(x)=1\}$ & 1 \\
\hline (V) & $\{ \pm 1\}$ & 1 \\
\hline
\end{tabular}

(4.8) In the table, the groups described are not the group $N$ itself but isomorphic images of $N$.

(4.9) For $t \in K^{\times}$, put $S_{Z}(t)=\left\{z \in Z=X \times X, q_{z}(z)=t\right\}$. Then, $h$ induces a map $h_{t}: S_{Z}(t) \rightarrow S_{W}\left(t^{2}\right)=\left\{w \in W=K \times X, q_{W}(w)=t^{2}\right\}$. Let $w \in S_{W}\left(t^{2}\right)$ be such that the fibre $h_{t}^{-1}(w) \neq \emptyset$. Since $z \in Z$ belongs to this fibre if and only if $h(z)=w$ and $q_{z}(z)=t$, the group $N$ acts on $h_{t}^{-1}(w)$. As $q_{z}(z)=n(x)+$ $n(y), q_{w}(w)=u^{2}+n(v), z=(x, y), w=(u, v)$, we have

$$
z \in h_{t}^{-1}(w) \Longleftrightarrow\left\{\begin{array} { l } 
{ n ( x ) + n ( y ) = t } \\
{ n ( x ) - n ( y ) = u } \\
{ 2 x y = v }
\end{array} \Leftrightarrow \left\{\begin{array}{l}
n(x)=r \\
n(y)=s, \\
2 x y=v
\end{array}\right.\right.
$$

with $r=\frac{1}{2}(t+u), s=\frac{1}{2}(t-u)$. Since $t \neq 0$, either $r$ or $s \neq 0$. If $r \neq 0$, then $x$ is invertible and $y=\frac{1}{2} x^{-1} v$. We have therefore the bijection $h_{t}^{-1}(w)$ $\approx\{x \in X, n(x)=r\}$. If we identify $N$ with $\{a \in X, n(a)=1, x(a z)=(x a) y\}$ by (4.1), then the action of $N$ on $h_{t}^{-1}(w)$ is given by $x \mapsto x a^{-1}$ or $x \mapsto a x$ according as $r \neq 0$ or $s \neq 0$. From the table of (4.7), we see that $N$ acts transitively on the fibre when $X$ is of type (II), (III), (IV). 


\section{§5. Tate-Shafarevich set for algebraic groups}

Let $k$ be an algebraic number field of finite degree over the field $\boldsymbol{Q}$ of rational numbers. Let $G$ be an algebraic group defined over $k$. Using the standard notation in Galois cohomology, we put

$$
\amalg(k, G)=\operatorname{Ker}\left(H^{1}(k, G) \longrightarrow \prod_{v} H^{1}\left(k_{v}, G\right)\right),
$$

and call this the Tate-Shafarevich set for $G$ over $k$. Basic references for Galois cohomology are [2] and [3]. In this section, we shall prove two lemmas which will be needed in the next section.

(5.1) Lemma. Let $K / k$ be a finite Galois extension and $G$ be an algebraic group defined over $k$. If $\amalg(K, G)=0$, then there is a bijection $\amalg(k, G) \approx$ $\amalg\left(K / k, G_{K}\right)$, where

$$
\amalg(K / k, G)=\operatorname{Ker}\left(H^{1}\left(K / k, G_{K}\right) \longrightarrow \prod_{v} H^{1}\left(K_{v} / k_{v}, G_{K_{v}}\right)\right)
$$

and $K_{v}$ is the field which is the completion of $K$ taken in the algebraic closure $\bar{k}_{v}$ of $k_{v}$.

Proof. Consider the following commutative diagram:

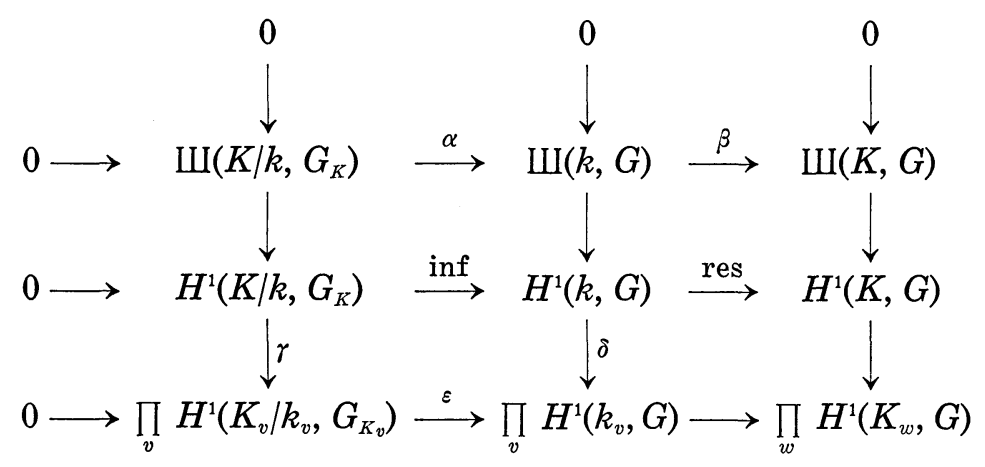

where all columns and the middle row are exact, $\alpha$, inf, $\varepsilon$ are injective and $K_{w}$ is the completion of $K$ at a place $w$ of $K$. We shall show that $\operatorname{Im} \alpha=\operatorname{Ker} \beta$. In fact, take $x \in \amalg\left(K / k, G_{K}\right)$. Then we have $\beta \alpha(x)=$ $\operatorname{res} \inf (x)=0$ and hence $\operatorname{Im} \alpha \subset \operatorname{Ker} \beta$. Next, take $y \in \operatorname{Ker} \beta \subset \operatorname{Ker}$ (res). Then $y=\inf (x)$ for some $x \in H^{1}\left(K / k, G_{K}\right)$. It then follows that $0=\delta(y)=$ $\delta \inf (x)=\varepsilon \gamma(x)$. Since $\varepsilon$ is injective, we have $\gamma(x)=0$, i.e. $x \in \amalg\left(K / k, G_{K}\right)$ which shows that $\operatorname{Ker} \beta \subset \operatorname{Im} \alpha$. Now, if $\amalg(K, G)=0$, then the relation $\operatorname{Im} \alpha=\operatorname{Ker} \beta$ means that $\alpha$ is surjective, which proves our assertion, q.e.d. 
(5.2) LEMma. We have $\amalg(k, G)=0$ if $G$ is a finite abelian group consisting of k-rational points only.

Proof. Denote by $\mathfrak{g}$ (resp. $\mathrm{g}_{v}$ ) the Galois group of $\bar{k} / k$ (resp. $\bar{k}_{v} / k_{v}$ ). By the assumption, $g$ and $g_{v}$ act trivially on $G$. Hence, $\amalg(k, G)$ is nothing else than the kernel of the canonical map

$$
\theta: \operatorname{Hom}(\mathfrak{g}, G) \longrightarrow \prod_{v} \operatorname{Hom}\left(g_{v}, G\right)
$$

where Hom means the continuous homomorphisms with respect to the Krull topology on the Galois group and the discrete topology on $G$. Now, take any $\xi \in \operatorname{Ker} \theta$. Because of the continuity of $\xi$, there is an open normal subgroup $\mathfrak{h}$ of $g$ such that $\xi(\mathfrak{h})=0$, and hence $\xi\left(\mathfrak{g}_{v} \mathfrak{h}\right)=0$ for all $v$. Call $K / k$ the finite Galois extension corresponding to $\mathfrak{h}$. To $\mathfrak{g}_{v} h$ corresponds the field $\left(K \cap k_{v}\right) / k$ which is the decomposition field of a valuation of $K$ which induces $v$ on $k$. For any $\sigma \in \mathfrak{g}$, put $s=\sigma \mathfrak{h} \in \mathfrak{g}(K / k)$, the Galois group of $K / k$. By Tschebotareff density theorem, one has $t s t^{-1} \in g\left(K / K \cap k_{p}\right)$ for some finite prime $\mathfrak{p}$ of $k$, and for some $t \in \mathfrak{g}(K / k)$. If one puts $t=\tau \mathfrak{h}$ with $\tau \in \mathfrak{g}$, then $\tau \sigma \tau^{-1} \in \mathfrak{g}_{p} \mathfrak{h}$. Hence $\xi(\sigma)=\xi\left(\tau \sigma \tau^{-1}\right)=0$ since $\xi \in \operatorname{Ker} \theta$, and so $\xi=0$, q.e.d.

\section{§6. Hasse principle for $G$ attached to a composition algebra}

Let $(X, n)$ be a composition algebra defined over a number field $k$. By definition, there is a composition algebra $\left(X_{k}, n_{k}\right)$ over $k$ such that $(X, n)$ is obtained by extending the ground field $k$ to a universal domain $\Omega$ containing $k$. The Hopf map

$$
h: Z=X \times X \longrightarrow W=\Omega \times X
$$

is given by $h(z)=(n(x)-n(y), 2 x y), z=(x, y)$, and the group $G$ of automorphisms of $h$ becomes an algebraic group defined over $k$. Our main result is the

(6.1) Theorem. Let $G$ be the group of automorphisms of the Hopf map associated to a composition algebra defined over a number field $k$. Then, we have $\amalg(k, G)=0$, i.e. the Hasse principle holds for $G$.

Proof. We split the proof into five cases according to the type of the algebra $\left(X_{k}, n_{k}\right)$ described in the theorem of Hurwitz.

Type (I). In this case $X=\Omega, n(x)=x^{2}$ and $h(z)=\left(x^{2}-y^{2}, 2 x y\right)$. By (2.6), (3.6) (or by a direct calculation) we see that $G$ is a finite abelian group consisting of elements $\pm 1, \pm r$ where 


$$
\gamma=\left(\begin{array}{rr}
0 & i \\
-i & 0
\end{array}\right), \quad i=\sqrt{-1}
$$

Put $K=k(i)$. Then, since every element of $G$ is $K$-rational, we have $\amalg(K, G)=0$ by (5.2). Thus $\amalg(k, G)=0$ if $i \in k$. On the other hand, in case where $i \notin k$, we have $\amalg(k, G) \approx \amalg\left(K / k, G_{K}\right)$ by (5.1). We now prove that $H^{1}\left(K / k, G_{K}\right)=0$ which, of course, implies that $\amalg\left(K / k, G_{K}\right)=0$. In fact, let $\left(a_{\sigma}\right)=\{1, a\}$ be a cocycle of $g(K / k)$ in $G_{K}$. This simply means that $a a^{s}=1$ where $s$ is the generator of $\mathrm{g}(K / k)$. One sees easily that $a= \pm 1$ and $1=1^{-1} 1^{s},-1=\gamma^{-1} \gamma^{s}$, which shows that $\left(a_{\sigma}\right)$ is trivial.

Type (II). In this case, from (4.7), it follows that

$$
0 \longrightarrow N \longrightarrow G \stackrel{\chi}{\longrightarrow}\{ \pm 1\} \longrightarrow 0 \quad \text { (exact) , }
$$

where $N \approx \Omega^{\times}$and hence $H^{1}(k, N)=0$ by Hilbert theorem 90. Take a cocycle $\left(a_{\sigma}\right)$ from $\amalg(k, G)$. Then, $b_{\sigma}=\chi\left(a_{\sigma}\right)$ defines a cocycle $\left(b_{\sigma}\right)$ in $\amalg(k,\{ \pm 1\})$ which is 0 by (5.2). This implies that $b_{\sigma}=1$, i.e. $a_{\sigma} \in N$. Since $H^{1}(k, N)=0$, we have $a_{o} \sim 0$ in $N$ and hence in $G$. We have thus proved that $\amalg(k, G)=0$.

Type (III). In this case, $X_{k}=K$, a quadratic extension, $n_{k}$ is the norm for $K / k$ and $N=\{x \in X, n(x)=1\}$, a torus of dimension one which is split by $K$. Hence, we have $H^{1}(K, N)=0$ by Hilbert theorem 90 . Take a cocycle $\left(a_{\sigma}\right)$ from $\amalg(K, G)$. Then $b_{\sigma}=\chi\left(a_{\sigma}\right)$ defines a cocycle $\left(b_{\sigma}\right)$ in $\amalg(K,\{ \pm 1\})$ which is 0 by (5.2). Hence $b_{o}=1$ and so $a_{\sigma} \in N$. Then, Hilbert theorem 90 for $N$ over $K$ implies that $a_{\sigma} \sim 0$ in $N$ and hence in $G$, which proves that $\amalg(K, G)=0$. Therefore, by (5.1), we have $\amalg(k, G) \approx \amalg\left(K / k, G_{K}\right)$. Having reduced the problem to the case of quadratic extension $K / k$, we write $K=k(\theta)$ with $\theta^{2}=m \in k$. Hence, we have $n(z)=x^{2}-m y^{2}$ if $z=x$ $+y \theta, x, y \in \Omega$. From (4.5), we see that, for any field extension $L / k$, we have $\left[G_{L}: N_{L}\right]=2$ if and only if $x^{2}-m y^{2}=-1$ has solutions $x, y$ in $L$. Now, take a cocycle $\left(a_{\sigma}\right)$ from $\amalg\left(K / k, G_{K}\right)$. By a similar argument as above, using $\amalg(K / k,\{ \pm 1\})=0$, we see that $a_{\sigma} \in N_{K}$. We shall prove that $a_{\sigma} \sim 0$ in $N_{K_{v}}$ for all $v$ in $k$. If that is so, our assertion will follow from the known fact $\amalg\left(K / k, N_{K}\right)=0$ (See [4] Proposition 4.5.1). First of all, if the valuation $v$ is such that $K_{v}=k_{v}$, then the matter is trivial because $\left(a_{\sigma}\right)=$ $\{1\}$. So, from now on, we shall only consider the case where $\left[K_{v}: k_{v}\right]=2$. In this case, we have $\left(a_{\sigma}\right)=\{1, a\}$ with $a \in N_{K}$. The assumption $a_{\sigma} \sim 0$ in $G_{K_{v}}$ means that $a=g_{v}^{-1} g_{v}^{s}$, where $s$ is the conjugation in $K_{v} / k_{v}$. If $g_{v} \in N_{K_{v}}$, then $a_{\sigma} \sim 0$ in $N_{K_{v}}$ already. If not, we write $g_{v}=w t_{v}$ with $t_{v} \in N_{K_{v}}$ and 
$w$, any element of $G_{K}-N_{K}$. We now show that we can find $w$ such that $w^{s}=-w$. Namely, identifying $X$ with $\Omega^{2}$ by setting $x_{1}+x_{2} \theta=\left(\begin{array}{l}x_{1} \\ x_{2}\end{array}\right)$, put $\beta(x)=\left(\begin{array}{ll}0 & \theta \\ \theta^{-1} & 0\end{array}\right)\left(\begin{array}{l}y_{1} \\ y_{2}\end{array}\right)$. Then, by (3.6), we see that $w=\left(\begin{array}{ll}0 & \beta \\ \beta & 0\end{array}\right) \in G_{K}-N_{K}$ because $n(\beta(y))=\left(\theta y_{2}\right)^{2}-m\left(\theta^{-1} y_{1}\right)^{2}=m y_{2}^{2}-y_{1}^{2}=-n(y)$ and $(\beta y)(\beta x)=\left(\theta y_{2}\right.$ $\left.+\theta\left(\theta^{-1} y_{1}\right)\right)\left(\theta x_{2}+\theta\left(\theta^{-1} x_{1}\right)\right)=m x_{2} y_{2}+x_{1} y_{1}+\theta\left(x_{1} y_{2}+x_{2} y_{1}\right)=\left(x_{1}+\theta x_{2}\right)\left(y_{1}+\theta y_{2}\right)$ $=x y$. It is obvious that $w^{s}=-w$. Using this $w$, we have $a=g_{v}^{-1} g_{v}^{s}=$ $t_{v}^{-1} w^{-1} w^{s} t_{v}^{s}=-t_{v}^{-1} t_{v}^{s}$. We next show that we can find $u \in N_{K_{v}}$ such that $-1=u^{-1} u^{s}$. In fact, take $\xi, \eta \in k_{v}$ such that $\xi+\eta=1, \xi-\eta=m^{-1}$, and put $x=\theta \xi, y=\eta, u=x+\theta y$. Then, $u^{s}=x^{s}-\theta y^{s}=-x-\theta y=-u$ and $n(u)=x^{2}-m y^{2}=m \xi^{2}-m \eta^{2}=m(\xi+\eta)(\xi-\eta)=1$. We have therefore $a=g_{v}^{-1} g_{v}^{s}=\left(t_{v} u\right)^{-1}\left(t_{v} u\right)^{s}$ and hence $a_{\sigma} \sim 0$ in $N_{K_{v}}$, again.

Type (IV). In this case, $G=N$ and $N=\{x \in X, n(x)=1\}$. Denote by $X_{0}$ the subspace of $X$ consisting of quaternions of trace zero and by $n_{0}$ the quadratic form induced on $X_{0}$ by $n$. Then $N$ is a simply connected group (the spin group) which forms a double covering of the rotation group $O^{+}\left(n_{0}\right)$. Then, we have $\amalg(k, G)=0$ as is well-known (See [3]).

Type (V). In this case, $G=N=\{ \pm 1\}$ and our assertion follows from (5.2), q.e.d.

\section{REFERENCES}

[1] R. Schafer, An introduction to nonassociative algebras, Academic Press, New York, 1966.

[2] J.-P. Serre, Cohomologie galoisienne, Lecture Notes in Mathematics, SpringerVerlag, 1964.

[ 3 ] M. Kneser, Lectures on Galois cohomology of classical groups, Tata Institute, Bombay, 1969.

[4] T. Ono, On the Tamagawa number of algebraic tori, Ann. of Math. 78 (1963), 47-73.

The Johns Hopkins University 